\title{
Généalogies des outils de gestion. Introduction
}

Management tools' genealogies. Introduction

Sarah Ghaffari, Séverine Misset, Fabienne Pavis et Marie Ponnet

\section{(2) OpenEdition}

Journals

Édition électronique

URL : http://journals.openedition.org/travailemploi/5889

DOI : 10.4000/travailemploi.5889

ISSN : $1775-416 \mathrm{X}$

Éditeur

DARES - Ministère du Travail

Édition imprimée

Date de publication : 15 mars 2013

Pagination : 5-12

ISSN : 0224-4365

\section{Référence électronique}

Sarah Ghaffari, Séverine Misset, Fabienne Pavis et Marie Ponnet, « Généalogies des outils de gestion.

Introduction », Travail et Emploi [En ligne], 133 | janvier-mars 2013, mis en ligne le 01 janvier 2013,

consulté le 22 septembre 2020. URL : http://journals.openedition.org/travailemploi/5889; DOI :

https://doi.org/10.4000/travailemploi.5889 


\title{
Généalogies des outils de gestion. Introduction
}

\author{
Sarah Ghaffari ${ }^{(*)}$, Séverine Misset ${ }^{(* *)}$, Fabienne Pavis ${ }^{(* *)}$, Marie Ponnet ${ }^{(* * *)}$
}

Une profusion d'outils de gestion est mise en œuvre dans les organisations produisant des biens et des services lucratifs ou non. On citera, par exemple, les démarches qualité, le Benchmarking, les Business plans, les normes environnementales, les grilles de rémunération, le coaching, la gestion prévisionnelle des compétences ou encore le suivi mensuel personnalisé des demandeurs d'emploi. Ces outils font l'objet de postures diverses : celle de théoriciens-praticiens cherchant in fine à optimiser les pratiques de rationalisation du travail; celle de chercheurs en sciences sociales voulant contrer les discours laudateurs sur la gestion et certaines dérives du management; ou encore celle visant à rendre compte des conditions de travail, actuelles ou passées, des salariés. Chacune de ces postures induit une manière spécifique de construire l'objet. Prendre le parti d'une approche généalogique des outils de gestion, c'est considérer que leur invention et leur mise en œuvre doivent être pensées en continuité. Ces outils, issus de conventions sociales, sont portés et modelés par des acteurs situés socialement, qui agissent dans des conjonctures spécifiques, sont intégrés à des logiques professionnelles et organisationnelles, sont impliqués dans des jeux d'alliance et de concurrence et sont porteurs de stratégies et de croyances. Ce constat incite à articuler une analyse interne aux outils centrée sur le technique et l'idéologique à une analyse externe, focalisée sur les acteurs et les organisations. En étudiant la filiation des outils de gestion et leurs développements successifs, les contributions de ce dossier déconstruisent ces universaux potentiellement puissants et coercitifs.

Précisons d'emblée le contour des outils de gestion qui nous intéressent ici. Il s'agit aussi bien des techniques de rationalisation concrétisées dans des instruments physiques (un indicateur du New Public Management, une technique de cotation au poste) que de dispositifs plus immatériels (une formation au développement personnel, le bénévolat d'entreprise) qui s'apparentent à des catégories

(*) CENS, École des mines de Nantes; sarah.ghaffari@minesnantes.fr

(**) CENS, Université de Nantes; severine.misset@univ-nantes.fr (***) IDHE, Université de Nantes; fabienne.pavis@univ-nantes.fr (****) LEMNA, École des mines de Nantes; marie.ponnet@ mines-nantes.fr d'action. Intégrer ces deux pôles permet de nourrir l'approche généalogique en saisissant la cristallisation d'une catégorie de pensée ou inversement la dématérialisation d'une technique. Ainsi, le management des compétences a été progressivement instrumenté par des référentiels s'appuyant sur des logiciels. À l'inverse, les démarches d'éco-conception, si elles se sont d'abord appuyées sur des bases de données, se diluent progressivement dans les discours sur la Responsabilité sociale de l'entreprise. Ce parti pris d'une définition extensive de l'objet invite également à déplacer le regard en posant à certains outils les questions généralement posées à d'autres. Croiser les approches souvent conçues exclusivement pour «la gestion» ou pour «le management» permet de se demander en quoi un outil tangible, comme l'indicateur hospitalier étudié par Nicolas Belorgey, est un mode de mobilisation de la main-d'œuvre ou inversement, en quoi un outil immatériel telle la formation «Entreprise de soi» prise pour objet par Hélène Stevens est un instrument qui impose une lecture standardisée du travail et des carrières. Enfin, ce choix a une implication en termes de vocabulaire. Si nous optons ici pour la nomination "outil de gestion» qui nous paraît la plus générique et la moins porteuse de paradigme théorique, a contrario de la notion foucaldienne de «dispositif» (Beuscart, Peerbaye, 2006), chacun des auteurs utilisera la terminologie la plus ajustée à son objet.

Réfléchir aux apports d'une approche généalogique des outils de gestion est l'occasion d'engager un dialogue entre les traditions intellectuelles qui permettent de les appréhender. Alors même qu'ils font l'objet de nombreuses études de sciences sociales, ces traditions communiquent peu entre elles. Cette introduction vise à mettre chacun des articles de ce dossier en perspective à l'aune de trois grilles de lecture (non exhaustives) susceptibles d'instruire une perspective généalogique ${ }^{(1)}$. La première grille, peut-être la plus immédiate, s'interroge sur les usages et l'appropriation des outils de gestion déployés au sein d'organisations contemporaines. Ce sont leurs développements successifs

(1) Ce dossier fait suite à la journée d'études «Sociologie des outils de gestion» organisée par le CENS en juin 2011 à l'Université de Nantes. Nos remerciements vont aux participants à cette journée ainsi qu'aux membres du comité de rédaction de Travail et emploi. 
associés à leurs conditions de circulation qui nourrissent l'approche généalogique. La deuxième grille d'analyse revient sur le processus sociotechnique de la création des standards et ce qu'il implique. Rendre compte de l'outil en train de se faire met l'approche généalogique au cœur de l'objet et plaide pour une compréhension des principes généraux qui la soustendent. La troisième grille de lecture se centre quant à elle sur des travaux afférents à la rationalisation industrielle. L'approche généalogique est alors soutenue par une réflexion sur les origines de ces outils au travers de la restitution du processus diachronique de leur implantation.

\section{Penser les usages et l'appropriation des outils de gestion au sein des organisations contemporaines}

La majorité des ouvrages et numéros spéciaux de revues consacrés à la sociologie des outils de gestion contemporains néglige leur dimension historique. À partir des années 1980-1990, une phase durant laquelle ces outils foisonnent est néanmoins identifiée en lien avec le développement des techniques informatiques, de nouvelles formes de mondialisation ou encore, la disqualification des collectifs (Maugeri, 2003; Terssac, Bazet, 2007). Dans le même temps, une série de travaux met en exergue la généralisation des outils de normalisation à l'ensemble des activités associées à l'extension du marché des services (GADREY, 1996; SEgRestin, 1997). En revanche, les rares enquêtes quantitatives sur le renouveau des outils de gestion sont peu convoquées, alors même qu'elles en mesurent la diffusion depuis vingt ans déjà (GREENAN et al, 2010). Et les apports de la tradition d'histoire et de sociologie des outils comptables et financiers sont négligés (CHIAPELLO, RAMIREZ, 2004). L'intérêt des travaux relevant de la sociologie des outils de gestion se situe donc ailleurs. Centrés sur les usages et les conditions d'appropriation de ces outils au sein des organisations contemporaines, ils décrivent, à partir d'observations synchroniques, avec des partis pris théoriques et méthodologiques contrastés, dans quelles circonstances, avec quelles intentions et quels effets, ils se diffusent. La dimension généalogique se réfère aux développements successifs de l'outil de gestion dans une temporalité courte. Dans cette perspective, l'outil prescrit - par le concepteur, le supérieur hiérarchique, les consultants - est nécessairement différent de l'outil réel, autrement dit celui utilisé par les acteurs en situation professionnelle, celui qui, pour être appréhendé, est modifié. Le standard se resocialise en quelque sorte dans sa configuration d'usage, comme l'illustrent dans ce numéro en particulier les articles d'Hélène Stevens et de Nicolas Belorgey, dont l'un des intérêts réside dans leur analyse de la chaîne d'acteurs qui investit l'outil au sein d'une institution.

Inscrits dans une tradition de sociologie des organisations, les travaux centrés explicitement sur les outils de gestion contribuent à la renouveler en l'associant aux apports de la sociologie des professions, du travail ou de l'entreprise (2). L'outil de gestion pris pour objet à l'échelle d'une organisation apparaît comme un potentiel actionné ou non, du fait de l'autonomie relative de logiques professionnelles, des rapports de force entre groupes, de la configuration sociale dans laquelle il est inséré (BOUSSARD, 2001). Co-construit, il est "travaillé», "modelé» par des groupes aux intérêts souvent contradictoires. Il peut ne pas être investi ou se trouver redéfini dans cette mise à l'épreuve (Rot, 2005; CochoY, Garel, Terssac, 1998; Reverdy, 2005). Hélène Stevens identifie ainsi les contournements dont la formation «Entreprise de soi» peut faire l'objet (l'investir comme thérapie et non comme dispositif de remobilisation dans le travail) et distingue les effets positifs des pratiques de réenchantement du travail offertes par le suivi de la formation, des effets négatifs de normalisation. Plus généralement, les effets coercitifs des outils de gestion sont interrogés (METZGer, Cleach, 2004 ; Chiapello, Gilbert, 2012).

D'inspiration plus anthropologique, d'autres travaux offrent une analyse fine, contextualisée et réflexive des conditions d'appropriation des instruments de rationalisation. La littérature managériale est décortiquée à partir de l'étude de la production et de la diffusion d'un journal d'entreprise (Вотн, 2006). Les logiques de fonctionnement contrastées de deux comités de direction sont restituées en particulier à partir de l'examen des réunions hebdomadaires (FLAMAND, 2002). Ou encore, les origines et les effets de l'irruption des normes qualité au sein d'un abattoir sont examinés (Muller, 2008). Les modalités d'enquête sont ici non seulement dévoilées mais utilisées comme source de connaissance. Les outils de gestion et leurs conditions de circulation peuvent également être appréhendés par les dispositions sociales que ceux-ci requièrent pour être utilisés. Ainsi, les logiques explicatives de leur diffusion ne s'arrêtent pas aux positions statutaires et professionnelles des acteurs au sein de l'organisation. Apparaissent les mécanismes sociaux de répulsion ou au contraire d'attraction ainsi que les effets inégalitaires de ces outils (VILlette, 1976; Pinto, 1987; Dassa, Maillard, 1996; Monchatre, 2011). Dans ce numéro, Nicolas Belorgey précise comment les trajectoires et ressources sociales contrastées des soignants éclairent l'emprise exercée par l'indicateur hospitalier qu'il étudie et en

(2) On situe ici la majorité des ouvrages collectifs centrés sur les outils de gestion sans restituer les oppositions "d'écoles» qui ne nous apparaissent pas toujours fondées (MAUGERI, 2001; Boussard, Maugeri, 2003; Boussard, 2005; Terssac, Bazet, RAPP, 2007). 
quoi, excluant les facteurs médicaux et sociaux, il est défavorable aux hôpitaux accueillant une population fragilisée. De même, Hélène Stevens montre que l'effet mobilisateur de la formation nécessite des ressources préalables inégalement réparties entre les individus et que l'outil constitue un vecteur de reproduction de l'ordre social. Il neutralise, rend invisibles des inégalités sociales (de classe, de sexe) et donc les reproduit, les durcit, les expulse du débat professionnel voire politique. Se situant à un niveau plus sociétal, Anne Bory révèle comment la rationalisation du mécénat par la promotion du bénévolat d'entreprise ne consiste pas seulement en un mode de mobilisation de la main-d'œuvre ou une réduction fiscale de plus : le développement de cette pratique bouleverse l'économie morale et financière du secteur associatif.

Enfin, l'étude de la population et des pratiques des professionnels de la rationalisation, qu'il s'agisse des ingénieurs des SSII (3) (ThINe, 2007), des graphologues (MARCHAL, 2005), des coachs, voire des chercheurs dans le cas de la psychodynamique (Salman, 2008), explore le rôle de la légitimité scientifique et technique dans les mécanismes d'appropriation des outils de gestion. Il existe un continuum de positions allant du praticien à l'académique avec toutes les positions intermédiaires de l'expertise et de la recherche-action (PAVIS, 2003). L'analyse lève, en outre, le voile sur un des ressorts de leur diffusion : la constitution et l'entretien d'un marché rentable (VIllette, 2003). Parce qu'ils sont bien souvent encastrés dans des dispositifs de régulation sociale, ils sont également façonnés par les organisations syndicales salariées (STEWART, 2006) et patronales, les branches, les territoires, et les dispositifs transnationaux (JoBerT, 2008; CAYET, 2010). La focale sur ces médiations juridiques et syndicales montre l'action déterminante des collectifs par exemple dans le processus d'individualisation, dans le cas d'entreprises ayant signé un accord sur la logique compétence (ZIMMERMANN, 2000). Dans la configuration particulière des années 1950, la contribution de Ferruccio Ricciardi qui examine tant le rôle des technocrates de la $\mathrm{CECA}^{(4)}$ et des experts du BIT ${ }^{(5)}$ que celui des syndicalistes, rend intelligibles les logiques de circulation et de traduction de cet instrument de régulation sociale qu'est la job evaluation. Son analyse a par ailleurs l'intérêt de revenir bien en amont de ce processus, en en proposant ainsi une déconstruction.

(3) Société de services en ingénierie informatique.

(4) Communauté européenne du charbon et de l'acier.

(5) Bureau international du travail.

\section{Penser la création des standards et ce qu'elle implique : rendre indiscutable le discutable}

Porteurs de modernité et d'efficacité, les outils de gestion, grâce à leur technicité et leur neutralité supposées se présentent comme les meilleures façons de maîtriser le fonctionnement des organisations. Leur inscription matérielle dans des productions techniques comme des codes, des standards, des labels ou des logiciels serait la garantie de leur neutralité et de leur puissance. Capables de rendre comparables des situations hétérogènes grâce à la stabilité de leurs indicateurs, ils seraient l'outil de pilotage par excellence. Loin de négliger ces présupposés, les sociologues des sciences et des techniques (au sens large) nous invitent au contraire à prendre pour objet les modalités de constitution des outils de gestion et à «ouvrir la boîte noire» de leur élaboration.

Dans cette deuxième grille de lecture, on s'intéresse à l'instrument en train de se faire et à son intrication dans des systèmes sociotechniques, associant des acteurs, des techniques et des interactions autour d'un objectif : la quantification de la valeur, indissociable de la rationalité économique, qu'il s'agisse de rendre compte de la valeur d'une entreprise ou d'une activité (VATIN, 2009). L'élaboration de critères retenus comme éléments fondant la bonne façon de juger met au jour des espaces de calcul en reconstruction et subséquemment des espaces de négociation potentiels. Dans le numéro, c'est ce à quoi s'attachent les articles de Nicolas Belorgey sur la construction d'un indicateur gestionnaire en milieu hospitalier et de Ferruccio Ricciardi sur la création d'un standard européen de qualification du travail et du travailleur dans le secteur sidérurgique.

Les concepts de traduction et de réseaux sociotechniques (AKRICH et al, 2006) se révèlent particulièrement opératoires pour analyser la genèse des outils de gestion. Plus qu'un simple choix technique ou organisationnel dans une palette préexistante, ils apparaissent, dans cette perspective, à la fois comme le résultat et le support de traductions. Ceci implique une sélection de la réalité sociale à analyser, des outils pour traduire cette sélection dans un langage partagé par les entités enrôlées dans le processus, et des réseaux pour diffuser et généraliser cette convention de mesure et l'objectiver dans des indicateurs et des procédures. Cette sélection et ce cadrage spécifient également les acteurs impliqués et leur rôle. Ils contribuent à échelonner les activités, les résultats et les personnes.

Cette déconstruction permet de voir comment la réalité sociale est "problématisée» selon certains points de vue et en fonction d'expertises variables, et comment ce questionnement est cadré par des modes d'observation et des choix d'indicateurs 
spécifiques. Cette posture est aussi celle qui alimente la sociologie de la quantification. Alain Desrosières montre que la construction d'un espace politique nécessite et rend possible l'émergence d'un «espace conventionnel d'équivalence» indiquant non seulement comment doit se faire la mesure mais aussi ce qui mérite d'être mesuré (DESROSIÈRES, 1993). Le langage pertinent est alors celui qui s'appuie sur un principe supérieur commun suffisamment partagé et généralisé pour en permettre une diffusion large (Boltanski, ThÉvenot, 1991). Partant de l'élaboration d'un indicateur dédié à quantifier le temps d'attente et de passage aux urgences, Nicolas Belorgey met au jour les justifications mobilisées et les traductions opérées pour l'inscrire dans un espace de communes mesures et lui conférer du sens, d'abord pour les gestionnaires et les soignants et ensuite, à travers d'éventuels palmarès publiés dans la presse, pour les patients. Un tel processus passe par l'élaboration et la (re)négociation de la convention susceptible de rendre comparable ce qui ne l'était pas d'emblée (KARPIK, 2007). Les critères communs sélectionnés pour saisir des réalités a priori hétérogènes, voire incommensurables, sont performatifs car ils énoncent les éléments qui doivent être pris au sérieux et, en creux, ceux qu'il convient d'occulter. La spécificité - et la puissance potentielle - de ces instruments est de tenir ensemble les logiques descriptive et prescriptive (Desrosières, 2008).

Ce travail de catégorisation concourt à la hiérarchisation sociale en différenciant la valeur des activités et des personnes qui y contribuent, voire en redéfinissant les attributs de la valeur. La généralisation des manuels dédiés au Business Plan standardise par exemple les projets de création d'entreprise. Via leurs check lists et leur feuille de route, ces ouvrages donnent les critères qualifiant le projet et permettent de les étalonner à l'aune des mêmes indicateurs (GIRAUDEAU, 2012). Disciplinant le regard, ils font d'une interprétation de la réalité la réalité, au motif que cette traduction s'étaie sur des principes «scientifiques» et des méthodes présentées comme objectives. Ferruccio Ricciardi expose ainsi comment la technique de job evaluation, d'une part est justifiée par la rigueur scientifique et l'équité de la méthode, et d'autre part contribue à standardiser les attributs de la qualité du travailleur par la cotation des postes. Donnant à voir des régularités réelles ou supposées, ces éléments combinés apparaissent comme des prises pour émettre un jugement (Bessy, Chateauraynaud, 1995) sur ce qu'est la bonne organisation des pratiques. Les compétences réunies dans un référentiel vont ainsi requalifier le bon travailleur. Le salarié voulant progresser s'appuiera sur les attributs énoncés pour orienter son action. Pour son évaluation, il devra s'assurer qu'il les interprète et les met en scène dans un langage intelligible pour son supérieur (BRETESCHE et al., 2010; Misset, 2009). Les outils de gestion sont à prendre comme des investissements de forme (THÉVENot, 1985) qui auraient en commun de s'appuyer sur un substrat technique (mesure, calcul, procédure, modèle), d'étayer une représentation simplifiée de l'organisation et de traduire une idéologie (Hatchuel, WeIL, 1992) telle que la rentabilité, la qualité ou la responsabilité sociale. Les analyser conduit à s'intéresser aux logiques politiques auxquelles ils sont associés dans leur mise en œuvre (Lascoumes, Le Galès, 2004). Reprenant l'idée que «le pouvoir dessine une mesure à sa mesure», certains auteurs poussent cette réflexion, en appréhendant la quantification comme une politique qu'il convient d'analyser en tant que telle (BARDET, JanY-CATRICE, 2010). La genèse de la technique de job evaluation traduit une volonté politique sur au moins deux plans : redéfinir les relations professionnelles et rendre comparable l'ensemble des salariés européens malgré leur situation nationale singulière. Cette convention d'équivalence générale favoriserait alors la circulation intra-européenne de la main-d'œuvre dans une logique marchande de concurrence pure et parfaite.

Mais progressivement, les conditions de genèse des instruments de quantification sont oubliées, ce qui en facilite la circulation dans des réseaux d'envergure variable. Cet enchaînement d'adaptations va durcir la réalité dont ils rendent compte. L'amnésie qui affecte les utilisateurs de ces outils favorise aussi la redéfinition des acteurs et des enjeux, sans que ceux qui sont mis en avant soient ceux qui étaient initialement visés. Un même outil peut soutenir des projets très différents selon les acteurs qui vont le mobiliser et les principes qu'ils vont y associer comme le montrent les déplacements opérés par l'outil de benchmarking (BRUNO, DIDIER, 2013). Cette autonomisation progressive donne tout son sens à l'analyse diachronique contextualisée.

\section{Penser la rationalisation industrielle : des méfaits du "taylorisme" à une sociohistoire des doctrines gestionnaires}

Centrées sur un processus historique clé, la transformation de l'industrie au tournant du $\mathrm{Xx}^{\mathrm{e}}$ siècle, des recherches inscrites dans une filiation de sociologie et d'histoire du travail ou de l'expertise ont l'intérêt de poser des bases de questionnements pour une sociohistoire de la rationalisation industrielle et des doctrines gestionnaires. Nous rapprochons donc, dans cette troisième grille de lecture, des travaux qui ne désignent également qu'exceptionnellement leur objet par le terme d' " outils de gestion», parlant plutôt d'《organisation», d' «administration», de «rationalisation». 
La sociologie du travail française s'est massivement construite en référence à la doctrine taylorienne et à son implantation supposée dans les entreprises. Lors de la seconde fondation de la discipline (Chapoulie, 1991), les recherches affiliées à Georges Friedmann ou Alain Touraine posent ainsi la question de l'évolution de la qualification face au progrès technique, à l'automatisation et au dépassement du taylorisme, dont on suppose qu'il aurait réduit «le travail en miettes» (FriedMANN, 1956). D'autres travaux (FreYsSENET, 1977; BAYART et al, 1984) continueront ensuite d'interroger l'évolution des qualifications face aux «nouvelles formes d'organisation du travail», alimentant un débat très nourri sur la déqualification ou la requalification des travailleurs et des collectifs de travail (PONNET, 2011), ou, plus largement, sur la caractérisation de la société comme néo - ou post - tayloriste. Hormis de rares travaux, comme celui de Bernard MotтEz (1966) sur les systèmes de rémunération, qui développe une critique d'une doctrine taylorienne aveugle aux rapports sociaux se jouant autour des salaires, cet ensemble de recherches n'interroge généralement pas plus le contenu de la doctrine que son implantation réelle, sa circulation ou son évolution. Autrement dit, seules sont interrogées les conséquences (les méfaits) d'un système qui n'est lui-même qu'indirectement questionné. François VATIN (1999) développera dès lors une critique de cette perspective d'appréhension de la rationalisation industrielle. Il estime en effet qu'en évacuant ces questions de généalogie, le «taylorisme» a finalement rarement été pris comme un objet «scientifique» mais plutôt comme une référence repoussoir. Exagérant l'impact et la diffusion du taylorisme, omettant l'analyse de la doctrine taylorienne (dont les conceptions n'étaient, selon lui, ni tellement novatrices, ni tellement fondées) et les critiques initiales dont elle a fait l'objet, la sociologie du travail aurait ainsi pris cet objet comme le symbole d'une conception rationalisatrice de la société, et en aurait paradoxalement assuré la postérité par ses critiques récurrentes.

À partir des années 1980, d'autres recherches (FridENSON, 1987) ont permis l'émergence d'une sociohistoire des doctrines gestionnaires (essentiellement le taylorisme, le fayolisme, le fordisme). L'origine des doctrines gestionnaires est retracée en lien avec les caractéristiques sociales et les parcours des fondateurs mais aussi avec ceux de leurs promoteurs : patrons techniciens (DENORD, Henry, 2007), ingénieurs modernistes comme Ernest Mattern (Cohen, 2001) ou Henry Le Chatelier (Henry, 2000), ministres promoteurs tel Étienne Clementel (FridENSON, 1987), sociétés de conseil (HENRY, 2012b). Quel système de croyances les anime? De quelles ressources bénéficient-ils pour faire circuler les idées et les techniques? Quelles luttes de concurrence permettent de comprendre les débats entre «rationalisateurs» et comment préciser l'état des forces opposées? La bataille Taylor / Fayol peut ainsi être vue comme la traduction d'une opposition naissante entre ingénieurs des fonctions de production et fonctions commerciales et financières. La position de Fayol requiert alors d'être replacée dans les débats de l'époque sur la méritocratie (HENRY, 2012a). Sont également restituées les conditions de constitution d'organisations patronales et étatiques (tels le CNOF, le COST, l'ITAP(6)) chargées de valoriser et diffuser ces modes de rationalisation (Moutet, 1997; KipPING, 2000).

Ce type de questionnement se révèle pertinent pour d'autres configurations historiques. Ferruccio Ricciardi, exploitant les archives de la CECA, étudie comment et pourquoi certains technocrates essaient de promouvoir la job evaluation, dresse un portrait des figures de promoteurs de la cotation au poste mais retrace aussi les débats internes autour de cet outil, dans le contexte de construction d'un marché commun du charbon et de l'acier. À l'échelle d'une formation d'entreprise, Hélène Stevens montre comment dans les années 1990, trois salariés aux profils contrastés ont pourtant conçu et promu ensemble «Entreprise de Soi»: un directeur des ressources humaines diplômé de droit des affaires et proche du Front national; un diplômé de sociologie, droit public et sciences politiques, proche de la gauche auto-gestionnaire; et une élue CGT au comité d'établissement. Cette genèse singulière éclaire les appropriations multiples dont cette formation «plurielle» peut alors faire l'objet. Enfin, Anne Bory remonte aux origines de la «référence américaine» puis retrace les jalons de l'importation en France d'un mécénat d'entreprise orienté non plus vers le domaine de la culture mais vers celui de la solidarité, le «bénévolat d'entreprise». Si elle évoque le rôle de certains promoteurs actifs de cette forme de bénévolat à l'instar de Claude Bébéar, créateur de l'Institut du mécénat de solidarité, elle met également en lumière certaines circonstances favorisantes comme les dispositifs fiscaux votés par différentes majorités.

Les recherches sur la rationalisation industrielle s'attachent aussi à identifier des facteurs favorables ou, au contraire, défavorables expliquant les conditions d'implantation des doctrines gestionnaires (Moutet, 1997; Boyer, 1983). À titre d'exemple, le contexte spécifique de la première guerre mondiale est souligné à maintes reprises comme facteur favorisant l'implantation de méthodes tayloriennes d'organisation du travail en facilitant la mise au travail de populations peu qualifiées, peu familiarisées avec l'univers industriel au moment où la main-d'œuvre qualifiée fait défaut et où les

(6) Comité national de l'organisation française; Centre d'organisation scientifique du travail; Institut technique de l'administration publique. 
besoins productifs sont maintenus à un haut niveau (FRIDENSON, 1987).

Mais ces recherches ne permettent pas toujours de voir fonctionner la rationalisation industrielle en acte, de cerner la divergence entre déclarations d'intentions et pratiques quotidiennes effectives (BOYER, 1983), et, lorsqu'elles cherchent à délimiter l'extension de l'implantation de ces doctrines gestionnaires, cèdent parfois au raisonnement en termes d'ortho - et d'hétérodoxie par rapport à une doctrine initiale, qui serait en quelque sorte «pure». Or une façon de dépasser le débat néo / post taylorien est de distinguer soigneusement discours, pratiques, organisation du travail et rapports sociaux pour évaluer l'ampleur des continuités et des ruptures dans le fonctionnement des entreprises contemporaines (LINHART, 1994). En analysant les conditions d'implantation de la job evaluation dans une usine pilote de Cornigliano en Italie, Ferruccio Ricciardi met en évidence que la convention négociée au niveau européen ne s'impose pas mais, au contraire, se superpose aux conventions locales et anciennes. Dès lors, articuler finement l'échelon local avec l'échelon global sur un temps suffisamment long, permet d'identifier à la fois comment les acteurs investissent les différentes médiations et comment ces médiations se construisent (Doublet, 1996; Moguen-Toursel, 2008). Cette posture permet en outre de s'interroger sur les relations existantes entre les acteurs et les outils de la rationalisation industrielle et ceux de la rationalisation administrative (Bezes, 2009; Bezes, Join-Lambert, 2010).

Les quatre articles présentés dans ce dossier «Généalogies des outils de gestion» empruntent, sans s'y laisser enfermer, aux trois grilles de lecture proposées ici sous forme de panorama intellectuel. Centrés sur des cas d'outils spécifiques, et à partir de matériaux empiriques diversifiés et ajustés à leur objet (archives, entretiens, observations, statistiques), ils contextualisent, historicisent, replacent les tensions qui entourent leur genèse, leur légitimation, leur appropriation, tout en laissant voir les acteurs qui se saisissent de ces enjeux. Ils ont la particularité de tenir ensemble, au moins partiellement, l'analyse interne et externe, un regard rétrospectif sur l'amont du processus de rationalisation et une attention simultanée à l'aval. Ce faisant, non seulement ils enrichissent la compréhension des objets étudiés mais alimentent une réflexion de portée plus générale. En revenant sur la genèse de la job evaluation, Ferruccio Ricciardi articule subtilement les échelons européen, national et local. Si dans tous les cas, la sélection des critères pertinents est un enjeu de mobilisation pour des acteurs aux intérêts divergents, l'une des forces de ce travail est de montrer que la clôture de la controverse se joue différemment selon le niveau observé. Cette posture offre donc une manière originale d'appréhender les ajustements nécessaires pour qu'un outil fasse sens dans un contexte autre que celui dans lequel il a été conçu. Nicolas Belorgey associe deux moments cruciaux et rarement étudiés de front de façon aussi fine : la construction du standard et son usage par les soignants. En déconstruisant un indicateur de gestion hospitalier, il montre en creux les conditions d'une possible subversion de l'outil : contrairement au chercheur, les acteurs de terrain n'ont ni le temps, ni les compétences, ni même l'intérêt professionnel, pour proposer un indicateur alternatif, qui ne serait pas focalisé sur les facteurs organisationnels. Hélène Stevens, à partir d'une formation suivie par $10 \%$ des salariés dans des conditions d'emploi qu'elle restitue, nous propose une analyse qui va des promoteurs de ce dispositif à leurs utilisateurs. En s'intéressant aux ressorts sociaux de l'enrôlement dans «Entreprise de soi», aux effets subjectifs et objectifs de cet outil de mobilisation de la main-d'œuvre à différents moments, elle en montre les faces cachées potentiellement transposables à de nombreux autres outils de gestion des ressources humaines, ou même d'accompagnement des demandeurs d'emploi. Enfin, Anne Bory nous fait découvrir les conditions de diffusion d'une nouvelle forme d'engagement au service de l'entreprise. Ici aussi, la dimension internationale, mobilisée pour mettre en perspective l'importation du bénévolat d'entreprise des États-Unis vers la France, ne se réduit pas à sa dimension comparative. Elle invite à saisir la genèse d'un outil à travers les traductions opérées et les réseaux mobilisés pour en favoriser la diffusion et la circulation hors de ses frontières initiales. 


\section{Bibliographie}

Akrich M., Callon M., Latour B. (2006), Sociologie de la traduction : textes fondateurs, Paris, Presses des Mines.

BARdet F., Jany-CATrice F. (2010), «Les politiques de quantification. Introduction au dossier », Revue française de socio-économie, $\mathrm{n}^{\circ}$ 5, pp. 9-17.

Bayart D., Berry M., Cavestro W. et al (1984), «Nouvelles technologies dans l'industrie : l'enjeu des qualifications », Sociologie du travail, $\mathrm{n}^{\circ} 4$.

Bessy C., Chateauraynaud F. (1995), Experts et faussaires : pour une sociologie de la perception, Paris, Métaillié.

Bezes P. (2009), Réinventer l'État. Les réformes de l'administration française (1962-2008), Paris, Presses universitaires de France.

Bezes P., Join-Lambert O. (2010), «Comment se font les administrations? Analyser les actes administratifs constituants», Sociologie du travail, vol. 52, $\mathrm{n}^{\circ} 2$, pp. 133-150.

Beuscart J.-S., Peerbaye A. (2006), «Histoires de dispositifs (introduction)», Terrains \& travaux, $\mathrm{n}^{\circ} 11$, pp. 3-15.

Boltanski L., Thévenot L. (1991), De la justification : les économies de la grandeur, Paris, Gallimard.

Вотн A. (2006), «Le journal interne d'entreprise. Ethnographie d'une mission impossible?», Ethnologie française, vol. 36, $\mathrm{n}^{\circ} 1$, pp. 45-54.

Boussard V. (2001), «Quand les règles s'incarnent. L'exemple des indicateurs prégnants», Sociologie $d u$ travail, vol. 43, n 4 , pp. 533-551.

Boyer R. (1983), «L'introduction du taylorisme en France à la lumière de recherches récentes. Quels apports et quels enseignements pour le temps présent?», Travail et emploi, $\mathrm{n}^{\circ} 18, \mathrm{pp} .17-41$.

Bretesché S., Ghaffari S., Kogan A.-F. (2010), «La démarche compétence pour requalifier le travail» in Bretesché S., Krohmer C. (dir), Fragile compétence, Paris, Presses des Mines, pp. 25-36.

Bruno I., Didier E. (2013), Benchmarking : l'État sous pression statistique, Paris, Zones.

CAYET T. (2010), Rationaliser le travail, organiser la production. Le BIT et la modernisation économique durant l'entre-deux-guerres, Rennes, Presses universitaires de Rennes.

Chapoulie J.-M. (1991), «La seconde fondation de la sociologie française, les États-Unis et la classe ouvrière», Revue française de sociologie, vol. 32, n 3, pp. 321-364.

Chiapello E., Ramirez C. (dir.) (2004), « Sociologie de la comptabilité», Comptabilité-Contrôle-Audit, $\mathrm{n}^{\circ} 10$.

Chiapello E., Gilbert C. (2012), «Les outils de gestion : producteurs ou régulateurs de la violence psychique au travail», Le travail humain, vol. 75, $\mathrm{n}^{\circ}$ 1, pp. 1-18.
Cochoy F., Garel J.-P., Terssac de G. (1998), « Comment l'écrit travaille l'organisation : le cas des normes ISO $9000 »$, Revue française de sociologie, vol. 39, $\mathrm{n}^{\circ} 4$, pp. 673-699.

Dassa S., Maillard D. (1996), «Exigence de qualité et nouvelles formes d'aliénation », Actes de la recherche en sciences sociales, $\mathrm{n}^{\circ} 115$, pp. 27-37.

Denord F., Henry O. (2007), «La "modernisation" avant la lettre : le patronat français et la rationalisation (1925-1940)», Sociétés contemporaines, n 68, pp. 83-104.

CoHen Y. (2001), Organiser à l'aube du taylorisme. La pratique d'Ernest Mattern chez Peugeot, 1906-1919, Besançon, Presses universitaires franc-comtoises.

Desrosières A. (2008), Pour une sociologie historique de la quantification, Paris, Presses des Mines.

Desrosières A. (1993), La politique des grands nombres. Histoire de la raison statistique, Paris, La Découverte, [2000].

Doublet J.-M. (coord.) (1996), «Les outils de gestion», Entreprises et histoire, $\mathrm{n}^{\circ} 13$.

Flamand N. (2002), Une anthropologie des managers, Paris, Presses universitaires de France.

FreysSenet M. (1977), La division capitaliste du travail, Paris, éd. Savelli.

FRIDENSON P. (1987), «Un tournant taylorien de la société française (1904-1918)», Annales. Économies, Sociétés, Civilisations, vol. 42, $\mathrm{n}^{\circ}$ 5, pp. 1031-1060.

Friedmann G. (1956), Le travail en miettes, Paris, Gallimard.

Gadrey J. (1996), Services, la productivité en question, Paris, Desclée de Brouwer.

Giraudeau M. (2012), «Remembering the future : entrepreneurship guidebooks in the US, from mediation to method (1945-1975)», Foucault Studies, n ${ }^{\circ}$ 13, pp. 40-66.

Greenan N., Guillemot D., Kocoglu Y. (coord.) (2010), «Informatisation et changements organisationnels dans les entreprises », Réseaux, vol. 28, n 162.

Hatchuel A., Weil B. (1992), L'expert et le système, Paris, Economica.

Henry O. (2012a), «Un entrepreneur de réforme de l'État : Henri Fayol (1841-1925)», Actes de la recherche en sciences sociales, $\mathrm{n}^{\circ} 193$, pp. 38-55.

Henry O. (2012b), Les guérisseurs de l'économie. Sociogenèse du métier de consultant (1900-1944), Paris, éd. CNRS.

Henry O. (2000), «Henry le Chatelier et le taylorisme», Actes de la recherche en sciences sociales, $\mathrm{n}^{\circ} 133$, pp. 79-88.

Jobert A. (dir.) (2008), Les nouveaux cadres du dialogue social - Europe et Territoires, Bruxelles, P.I.E. Peter Lang. 
KARPIK L. (2007), L'économie des singularités, Paris, Gallimard.

KIPPING M. (coord.) (2000), «Les consultants», Entreprises et histoire, $\mathrm{n}^{\circ} 25$.

Lascoumes P., Le Galès P. (2004), Gouverner par les instruments, Paris, Presses de Sciences Po.

LinHART D. (1994), La modernisation des entreprises, Paris, La Découverte, [2004, 2e éd.].

Marchal E. (2005), «Graphologie et entreprises : histoire et controverses », Sociologies pratiques, $\mathrm{n}^{\circ} 10$, pp. 57-76.

MAUGERI S. (2003), «Une lecture pessimiste du processus de rationalisation. L'exemple des dispositifs de gestion logistique», in Boussard V., Maugeri S., Du politique dans les organisations. Sociologies des dispositifs de gestion, Paris, L'Harmattan, pp. 105-132.

Maugeri S. (dir.) (2001), Délit de gestion, Paris, La Dispute.

Metzger J.-L., Cléach O. (2004), «Le télétravail des cadres : entre suractivité et apprentissage de nouvelles temporalités», Sociologie du travail, vol. 46, $\mathrm{n}^{\circ} 4$, pp. 433-450.

Moguen-Toursel M. (coord.) (2008), «Les entrepreneurs de standards », Entreprises et histoire, $\mathrm{n}^{\circ} 51$.

Muller S. (2008), À l'abattoir. Travail et relations professionnelles face au risque sanitaire, Paris, éd. MSH.

Misset S. (2009), La déstabilisation des strates qualifiées du groupe ouvrier. Le cas des ouvriers professionnels de Peugeot-Citroën, thèse de doctorat, sociologie, Université Paris Descartes.

Monchatre S. (2011), «Ce que l'évaluation fait au travail. Normalisation du client et mobilisation différentielle des collectifs dans les chaînes hôtelières », Actes de la recherche en sciences sociales, $\mathrm{n}^{\circ} 189$, pp. 43-57.

Mottez B. (1966), Systèmes de salaire et politiques patronales. Essai sur l'évolution des pratiques et des idéologies patronales, Paris, éd. CNRS.

Moutet A. (1997), Les logiques de l'entreprise. La rationalisation dans l'industrie française de l'entredeux-guerres, Paris, éd. EHESS.

Pavis F. (2003), Sociologie d'une discipline hétéronome. Le monde des formations en gestion entre universités et entreprises en France, 1960-1990, thèse de doctorat, sociologie, Université de Paris Panthéon-Sorbonne.

PINTO L. (1987), "Graphique et science d'entreprise», Actes de la recherche en sciences sociales, $\mathrm{n}^{\circ}$ 69, pp. 93-97.

Ponnet M. (2011), Les relations de sous-traitance et leurs effets sur la sûreté et la sécurité dans deux entreprises :
SNCF et $G r D F$, thèse de doctorat, sociologie, Université de Nantes.

REVERDY T. (2005), «Trajectoires de progiciels, trajectoires professionnelles », in Boussard V. (dir.), $A u$ nom de la norme : les dispositifs de gestion entre normes organisationnelles et normes professionnelles, Paris, L'Harmattan, pp. 203-225.

Rot G. (2005), «Le Knowledge Management et l'économie du partage des connaissances. Propos sur un désinvestissement de forme», Économies et sociétés, Série socio-économie du travail, $\mathrm{n}^{\circ} 25, \mathrm{pp}$. 675-698.

Salman S. (2008), «Fortune d'une catégorie : la souffrance au travail chez les médecins du travail», Sociologie du travail, vol. 50, $\mathrm{n}^{\circ}$ 1, pp. 31-47.

Segrestin D. (1997), «L'entreprise à l'épreuve des normes de marché - Les paradoxes des nouveaux standards de gestion dans l'industrie», Revue française de sociologie, vol. 38, $\mathrm{n}^{\circ} 3$, pp. 553-585.

Stemart P., Durand J.-P., Lewchuk W., Yates C., DANFORD A. (2006), "Les ouvriers de Vauxhall face à la lean production», Le mouvement social, $\mathrm{n}^{\circ} 217$, pp. 33-52.

Terssac G. de, Bazet I. (2007), "Les TIC-TAC de la rationalisation : un travail d'organisation?», in Terssac G. de, Bazet I., Rapp. L. (dir.), La rationalisation dans les entreprises par les technologies coopératives, Toulouse, Octarès, pp. 15-27.

Thévenot L. (1985), «Les investissements de forme», in Eymard-Duvernay F., Thévenot L. (dir), Conventions économiques, Les Cahiers du CEE, $\mathrm{n}^{\circ} 29$, Paris, Presses universitaires de France, pp. 21-71.

THINE S. (2007), «Redistribution des rôles dans l'urgence du déploiement de l'ERP. Un projet entre tutelle des consultants et prescription du marché», in Terssac G. de, Bazet I., Rapp. L. (dir), La rationalisation dans les entreprises par les technologies coopératives, Toulouse, Octarès, pp. 95-105.

VATIN F. (dir.) (2009), Évaluer et valoriser. Une sociologie économique de la mesure, Toulouse, Presses universitaires du Mirail.

VATIN F. (1999), Le travail, sciences et société. Essais d'épistémologie et de sociologie du travail, Bruxelles, Éd. de l'Université de Bruxelles.

Villette M. (2003), Sociologie $d u$ conseil en management, Paris, La Découverte.

Villette M. (1976), «Psychosociologie d'entreprise et rééducation morale», Actes de la recherche en sciences sociales, vol. 2, n 4, pp. 47-65.

Zimmermann B. (2000), «Logiques de compétences et dialogue social», Travail et emploi, $\mathrm{n}^{\circ}$ 84, pp. 5-18. 